\title{
Preliminary evidence-based recommendations for return to learn: a novel pilot study tracking concussed college students
}

\author{
Zachary W Bevilacqua', Mary E Kerby ${ }^{2}$, David Fletcher ${ }^{3}$, Zhongxue Chen ${ }^{4}$, Becca Merritt ${ }^{1}$, \\ Megan E Huibregtse ${ }^{1}$ \& Keisuke Kawata*,1,5 \\ ${ }^{1}$ Department of Kinesiology, School of Public Health, Indiana University, Bloomington, IN 47405, USA \\ ${ }^{2}$ Western University College of Osteopathic Medicine of the Pacific-Northwest, Lebanon, OR 97355, USA \\ ${ }^{3}$ Indiana University Health Center, Indiana University, Bloomington, IN 47405, USA \\ ${ }^{4}$ Department of Epidemiology \& Biostatistics, School of Public Health, Indiana University, Bloomington, IN 47405, USA \\ ${ }^{5}$ Program in Neuroscience, College of Arts \& Sciences, Indiana University, Bloomington, IN 47405, USA \\ *Author for correspondence: Tel.: 812855 5244; kkawata@indiana.edu
}

\begin{abstract}
Aim: Students re-entering the academic setting after a concussion is commonly referred to as return-tolearn and, to date, very few studies have examined the return-to-learn aspect of concussion recovery. Methodology: Nine college-aged, full-time students who were diagnosed with concussions were monitored throughout their concussion recovery. The severity for five chief symptoms (headache, dizziness, difficulty concentrating, fatigue, anxiety) were recorded six-times per day through text messages, and daily phone calls recorded participant's behavioral traits. Results: We identified five behavioral variables which significantly influenced symptom resolution (music, sleep, physical activity, water and time) ( $p=0.0004$ to $p=0.036$ ). Additionally, subjects reported math and computer-oriented courses as the most difficult (33 and 44\%, respectively). Conclusion: We introduce a novel approach to monitor concussed students throughout their recovery, as well as factors that may influence concussion recovery process.

Lay abstract: Concussed students and student-athletes returning to the classroom is commonly referred as return-to-learn. To date, there are very few recommendations that can aid students' concussion recovery in relation to their academic responsibilities. This is trouble giving that every student-athlete is first and foremost a 'student'. In light of this, we sought to track behavioral factors that might influence the resolution of college students' concussion symptoms as they recover from their injury and return to their coursework. Students also gave a perception of their recovery, including what types of activities and school accommodations appeared beneficial. In turn, behaviors such as minimizing music, getting more sleep, introducing more physical activity, drinking more water and overall time since injury appeared to help resolving the concussion symptoms. Additionally, students reported that math and computer-oriented courses were the most difficult, and that additional time on assignments/exams and reducing screen brightness were the most beneficial accommodations. These results are the first of their kind and will optimistically lead to recommendations utilized by healthcare professionals.
\end{abstract}

First draft submitted: 29 May 2019; Accepted for publication: 18 July 2019; Published online: 20 September 2019

Keywords: concussion $\bullet$ longitudinal $\bullet \mathrm{mTBI} \bullet$ phone call • recommendations $\bullet$ return to classroom • return to school • return-to-learn $\bullet$ RTL $\bullet$ text message

In 2012, The Atlantic listed concussion as one of the top ten ideas that changed the world [1]. The spotlight continued with the New York Times' print of '110 NFL Brains', highlighting Dr Anne McKee and her team's work on deceased American football players [2]. As the magnifying glass grows on concussion and our focus sharpens, it is becoming ever more important to holistically examine concussion, rather than solely focusing on treating brain injury in sport. Emerging evidence has given way to protocols to ensure the safe return of athletes to sport settings [3];

Future Medicine 
however, every concussed athlete at university is first and foremost a student-athlete, with 'student' holding the emphasis, which supports the need for better post-concussion management in academic settings. Furthermore, it is important to be inclusive of nonathlete patients who have been previously excluded from return-to-learn (RTL) literature [4-15].

A student re-entering the academic setting after a concussion is commonly referred to as RTL. To date, very few studies have examined the RTL aspect of concussion recovery and the effects that premature classroom attendance may cause. For instance, Ransom et al. demonstrated that concussions can negatively impact one's perception toward academic tasks (e.g., difficulty in taking notes, studying and understanding class materials). Difficulties in perception toward linguistic and math/science classes were age-dependent, with a larger portion of high school students expressing their concern in these classes compared with their middle school and elementary school counterparts [16]. In addition, as many as $45 \%$ of concussed students may be re-entering the classroom prematurely, as defined by a recurrence or exacerbation of symptoms following after returning to the classroom [17]. Only a handful of opinion-based recommendations have been given to alleviate the worsening of concussion symptoms, such as educating academic personnel about concussion recovery and prompt initiation of academic adjustments [18]. While these preliminary studies have provided initial evidence, these data are derivative of cross-sectional studies and retrospective chart review findings.

Until now, little has been done to investigate the factors influencing safe return to academics, as evident by the scarcity of consensual evidence-based recommendations [19]. Consequently, only nine US states (IL, MA, ME, MD, NE, NY, OK, VA, VT) require RTL implementation [20]. Review of RTL literature indicates several gaps in knowledge, with disagreement among RTL protocols ranking as a chief concern [19]. The state of RTL is relatively stagnant perhaps due to the absence of sound methodology that can provide holistic evidence-based results. Therefore, we have developed a novel method for comprehensively monitoring concussed students throughout their recovery and full return to academic participation. There are two aims in this paper. First, we longitudinally investigated the potential factors influencing resolution of chief concussion symptoms in college students who were diagnosed with concussions. Second, we assessed participants' perception of their concussion recovery and identified what types of activities and accommodations appeared beneficial.

\section{Methods}

\section{Participants}

Nine young adults volunteered to participate in this study. The study consisted of four male and five females with an average age of $20.2 \pm 1.6$ years. Inclusion criteria included being between 18 and 26 years of age, being enrolled at the studied University full time (undergraduate: 12 credit hours, graduate: 6 credit hours), possessing a cellular phone with text message and phone call capabilities and having received a diagnosis of concussion within 7 days of injury by the same sports medicine trained physician at the studied University Health Center, who was blinded from any data related to this study. Exclusion criteria included any head, neck or face injury 1 year prior to the study (e.g., concussion, eye injury); history of vestibular, ocular or vision dysfunctions (e.g., macular degeneration); pregnancy; any neurological disorders and any sleep disorders (e.g., sleep apnea, insomnia). All subjects gave written informed consent, and the studied University institutional review board approved the study.

\section{Study procedure}

Upon referral from the sports medicine physician, participants met with a member of the research team to review their eligibility. Each participant was fitted with an ActiGraph wrist watch (ActiGraph wGT3X-BT) and was instructed to wear the watch during the entirety of their participation in the study, except when bathing [21]. Participants were asked to complete a Qualtrics survey (Qualtrics International Inc., Provo, UT, USA) examining their demographic information as well as previous and current medical and academic history. Some of the information gathered from this initial survey pertains to the participant's pre-injury levels (e.g., symptom scores). These data were used as the participants' recovery criteria (see 'Recovery criteria' section). In an effort to limit participant's exposure to screens, the questions of this survey were asked verbally by the researcher. Next, the researcher performed a test-run to ensure that each participant's cellular phone was able to receive and respond to both a text message and phone call. Once confirmed, participants were told that they would begin receiving text messages on the same day, and their first daily phone call should be expected the following day. Refer to Figure 1 for procedural timeline. 


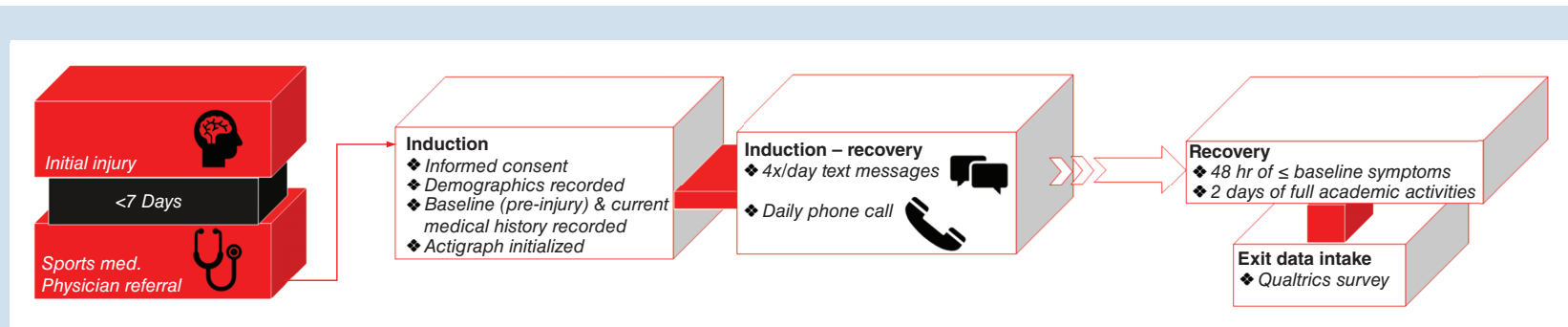

Figure 1. Study procedure timeline.

\section{Activity \& sleep measures}

ActiGraph data were analyzed using ActiLife (version 6.5.3) software, and we employed the Sadeh algorithm to score activity level via step count and sleep duration. Sleep duration detected by the ActiGraph has been validated with 91.4-96.5\% minute-by-minute agreement rates in adults compared with traditional polysomnography [21,22]. Data were scored in 60-s epochs as 'wake' or 'sleep' by measuring movement using triaxial accelerometer during each epoch, as well as movement immediately prior to and after each epoch. Subjective sleep log was used to visually inspect the ActiGraph data. Our primary interest of the total 24-h sleep (night sleep + naps) from each day until recovery was used for the statistical analysis. Similarly, we extrapolated step counts in 60-s epochs to assess activity levels, and the total step counts from each day until recovery was used for the analysis.

\section{Text message responses}

These messages were delivered to participants at four-timepoints (9 AM, 1 PM, 5 PM, 9 PM) each day. The text messages were used to collect symptom severity of five different symptoms on a $0-10$ scale, with 0 representing absence of the symptom and 10 representing the most severe level of the symptom. The text messages read as follows: "Please rate your current symptoms. Headache, Dizziness, Difficulty Concentrating, Fatigue, Anxiety". If a participant did not answer the text message promptly (e.g., they were asleep, or in class), they were instructed to respond as soon as possible. These five particular symptoms were chosen based on their high prevalence postconcussion (headache, dizziness, difficulty concentrating, fatigue), [23,24] or among the population (anxiety) [25]. These symptoms captured the three symptom categories of concussion; physical, cognitive and emotional [23,24].

\section{Phone call responses}

Phone calls were made to participants daily, at the same time (6 PM) by the same member of the research team. These calls were used to collect data regarding participant's diet (e.g., type of nutrient intake, number of meals, water/caffeine/alcohol consumption), duration of screen-time usage, music listened to, physical activity participation, medication/substance (NSAID or marijuana) usage, types of classes attended and alteration in symptom severity during the attended classes. For full list of questions, please refer to Supplementary Material 1. The current manuscript focuses our preliminary data on an association between selected behavioral factors and symptomatology; hence, all dietary factors or medication associations are not included in this manuscript. If a participant did not answer the daily phone call, they were instructed to call the researcher back as soon as possible. In case of nonrespondence, a second attempt to reach the participant was made at around 9 PM. The same researcher made each phone call based on a script. Phone call data were used in conjunction with text message data to determine recovery status, described in detail below.

\section{Recovery criteria}

The present study utilizes diagnosis of concussion from an independent sports medicine physician as inclusion criteria; however, we did not use medical clearance from this physician as our recovery criteria. By using text message symptom data, in addition to phone call class attendance data, we were able to satisfactorily determine 'full recovery'. Specifically, participants were required to meet the following criteria to be deemed recovered, maintain symptom levels at-or-below their individual baseline for a 48-h period (eight consecutive text messages), and attend their regular class schedule for a 2-day period without an increase in symptomatology beyond baseline. The rationale for this recovery criteria is as follows: first, not all students in attendance at the studied university 
Table 2. Behavioral variables - symptom severity associations.

\begin{tabular}{|c|c|c|c|c|c|c|c|c|c|c|}
\hline \multirow{2}{*}{$\begin{array}{l}\text { Behavioral } \\
\text { variables }\end{array}$} & \multicolumn{2}{|c|}{ Headache } & \multicolumn{2}{|c|}{ Dizziness } & \multicolumn{2}{|c|}{ Difficulty concentrating } & \multicolumn{2}{|c|}{ Fatigue } & \multicolumn{2}{|c|}{ Anxiety } \\
\hline & Estimate (SE) & p-value & Estimate (SE) & p-value & Estimate (SE) & $p$-value & Estimate (SE) & $p$-value & Estimate (SE) & p-value \\
\hline $\begin{array}{l}\text { Time: hour } \\
\text { postinjury }\end{array}$ & $\begin{array}{l}-0.00332 \\
(0.00121)\end{array}$ & $0.0064^{\dagger}$ & $\begin{array}{l}-0.00173 \\
(0.00072)\end{array}$ & $0.0174^{\dagger}$ & $\begin{array}{l}-0.00295 \\
(0.00159)\end{array}$ & 0.0647 & $\begin{array}{l}-0.00409 \\
(0.00135)\end{array}$ & $0.0026^{\dagger}$ & $\begin{array}{l}-0.00412 \\
(0.00141)\end{array}$ & $0.0038^{\dagger}$ \\
\hline Step count & $\begin{array}{l}0.00005 \\
(0.00004)\end{array}$ & 0.2177 & $\begin{array}{l}-0.00004 \\
(0.00002)\end{array}$ & 0.0646 & $\begin{array}{l}0.00005 \\
(0.00005)\end{array}$ & 0.2448 & $\begin{array}{l}0.00005 \\
(0.00004)\end{array}$ & 0.2923 & $\begin{array}{l}0.00006 \\
(0.00004)\end{array}$ & 0.1336 \\
\hline Sleep (min) & $\begin{array}{l}0.00013 \\
(0.00033)\end{array}$ & 0.7023 & $\begin{array}{l}-0.00064 \\
(0.00018)\end{array}$ & $0.0004^{\ddagger}$ & $\begin{array}{l}-0.0002 \\
(0.00038)\end{array}$ & 0.5995 & $\begin{array}{l}-0.00032 \\
(0.00038)\end{array}$ & 0.3911 & $\begin{array}{l}-9.11 \mathrm{E}-6 \\
(0.00033)\end{array}$ & 0.9781 \\
\hline $\begin{array}{l}\text { Water intake } \\
(8 \mathrm{oz})\end{array}$ & $\begin{array}{l}0.04689 \\
(0.04407)\end{array}$ & 0.2881 & $\begin{array}{l}-0.04188 \\
(0.02573)\end{array}$ & 0.1045 & $\begin{array}{l}0.06481 \\
(0.05607)\end{array}$ & 0.2485 & $\begin{array}{l}0.01833 \\
(0.04944)\end{array}$ & 0.7111 & $\begin{array}{l}-0.1719 \\
(0.04939)\end{array}$ & $0.0006^{\ddagger}$ \\
\hline $\begin{array}{l}\text { Caffeine } \\
\text { intake (oz) }\end{array}$ & $\begin{array}{l}-0.00644 \\
(0.02438)\end{array}$ & 0.7917 & $\begin{array}{l}-0.01031 \\
(0.01365)\end{array}$ & 0.4507 & $\begin{array}{l}-0.01214 \\
(0.02932)\end{array}$ & 0.6790 & $\begin{array}{l}-0.03136 \\
(0.02751)\end{array}$ & 0.2552 & $\begin{array}{l}-0.04795 \\
(0.02560)\end{array}$ & 0.0619 \\
\hline $\begin{array}{l}\text { Screen time } \\
(\min )\end{array}$ & $\begin{array}{l}-0.00279 \\
(0.00170)\end{array}$ & 0.1025 & $\begin{array}{l}0.00004 \\
(0.00095)\end{array}$ & 0.9644 & $\begin{array}{l}-0.00368 \\
(0.00205)\end{array}$ & 0.0730 & $\begin{array}{l}-0.00082 \\
(0.00192)\end{array}$ & 0.6716 & $\begin{array}{l}0.00022 \\
(0.00179)\end{array}$ & 0.9014 \\
\hline Music (min) & $\begin{array}{l}0.00914 \\
(0.00264)\end{array}$ & $0.0006^{\ddagger}$ & $\begin{array}{l}-0.00064 \\
(0.00152)\end{array}$ & 0.6714 & $\begin{array}{l}0.00806 \\
(0.00329)\end{array}$ & $0.0149^{\dagger}$ & $\begin{array}{l}0.007180 \\
(0.00297)\end{array}$ & $0.0160^{\dagger}$ & $\begin{array}{l}0.00535 \\
(0.00289)\end{array}$ & 0.0650 \\
\hline $\begin{array}{l}\text { Physical } \\
\text { activity } \\
\text { (absence of) }\end{array}$ & $\begin{array}{l}0.6621 \\
(0.3236)\end{array}$ & 0.0867 & $\begin{array}{l}0.2468 \\
(0.1798)\end{array}$ & 0.2189 & $\begin{array}{l}1.0347 \\
(0.3852)\end{array}$ & $0.0362^{\dagger}$ & $\begin{array}{l}1.5307 \\
(0.3658)\end{array}$ & $0.0058^{\dagger}$ & $\begin{array}{l}0.4302 \\
(0.3360)\end{array}$ & 0.2477 \\
\hline \multicolumn{11}{|c|}{$\begin{array}{l}\dagger * p<0.05 \\
\ddagger * \star p<0.001 . \\
\text { Data are displayed as estimate values with accompanying SEs. Level of significance set at } p<0.05 \text {. } \\
\text { SE: Standard error of mean. }\end{array}$} \\
\hline
\end{tabular}

carry equal levels of health insurance coverage, therefore the financial burden due to follow-up physician visits may discourage some students from returning. Second, a recent systematic review showed that concussion literature varied in their definitions of what constituted 'full recovery'. Across the 43 included articles, $100 \%$ used a return to baseline somatic symptoms as recovery criterion, $86 \%$ used return to baseline neurocognitive testing scores, $49 \%$ used a return to physical exertion with no symptom exacerbation, only $30 \%$ included normalization of balance [26], indicating that symptom resolution is the most consistent criteria for full recovery among concussion literature. Third, a systematic review by Purcell et al. [9] indicates that several studies stress the need for effective communication between educators and practitioners. Therefore, it was unlikely the diagnosing physician would receive feedback from the participants' instructors as to their academic performance or attendance. In turn, we sought to establish a conservative set of criteria that would provide us with data indicating that students had achieved a symptom-free state, in addition to having returned to their course load without symptom exacerbation.

\section{Exit (post-recovery) data intake}

Once a status of full recovery was deemed, a member of the research team met with participants for an exit interview. This interview consisted of collecting the ActiGraph wristwatch and completion of an exit survey. This exit survey asked participants about their perceptions and experiences during their time enrolled in the study. For full list of exit interview questions, please refer to Supplementary Material 2.

\section{Statistical analysis}

Descriptive statistics were used to analyze the demographic information and methodological efficacy, as measured by the Qualtrics survey and phone call/text message response rates, respectively. To explore the factors influencing five symptoms, we conducted a mixed-effects regression models (MRM). The model included five symptoms as outcome variables with eight independent variables; time, step count, sleep, water intake, caffeine intake, screen time, music and physical activity. The MRM was used to accommodate both repeated measurements within each day, as well as between days throughout recovery. Independent variables are expressed in different units as explained in Table 2. A positive estimate value indicates a worsening or increase in the symptom, whereas a negative estimate value indicates a resolving effect on the symptom. All MRM analyses were conducted using SAS statistical software and the level of significance was set at $\mathrm{p}<0.05$. Data for the exit survey were descriptively presented as percentages of participants that selected a given answer. 
Table 1. Demographics.

\begin{tabular}{|c|c|}
\hline Gender & 5 female: 4 male \\
\hline Age & $20.2 \pm 1.6$ years \\
\hline Body mass index & $24.8 \pm 3.0 \mathrm{k} / \mathrm{m}^{2}$ \\
\hline \multicolumn{2}{|l|}{ Hx of physician treatment for } \\
\hline Migraines & No $(89 \%)$ : yes $(11 \%)$ \\
\hline Epilepsy or seizures & No $(100 \%)$ \\
\hline Substance or alcohol abuse & No $(100 \%)$ \\
\hline Psychiatric conditions & No $(56 \%)$ : yes $(44 \%)$ \\
\hline Diagnosed with ADD/ADHD & No $(67 \%)$ : yes $(33 \%)$ \\
\hline Diagnosed with HIV & No $(100 \%)$ \\
\hline Number of previous concussions & $0.6 \pm 1.0$ \\
\hline Time of current injury & Morning $=3 ;$ afternoon $=5 ;$ evening $=1$ \\
\hline Location of impact & Frontal = 5; parietal = 2; temporal = $3 ;$ occipital $=3$ \\
\hline Cause of injury & Sport $=2 ;$ motor vehicle accident $=3 ;$ fight $=2 ;$ fall $=3$ \\
\hline Days till recovered & $18.3 \pm 7.7$ (Min: 10 to Max: 36$)$ \\
\hline \multicolumn{2}{|l|}{ Pre-injury symptom levels } \\
\hline Headache & $0.9 \pm 1.5$ \\
\hline Fatigue & $1.6 \pm 1.3$ \\
\hline Dizziness & 0 \\
\hline Difficulty concentrating & $1.2 \pm 1.9$ \\
\hline Anxiety & $2.3 \pm 2.6$ \\
\hline Total pre-injury symptom score & $6 \pm 4.7$ \\
\hline \multicolumn{2}{|l|}{ Pre-injury behaviors: } \\
\hline Hours of sleep per night & $7 \pm 4.8$ \\
\hline Minutes listening to music & $143 \pm 92$ \\
\hline Minutes of screen time & $373 \pm 182$ \\
\hline Caffeine intake & Always $=1 ;$ often $=2 ;$ sometimes $=3 ;$ rarely $=2 ;$ never $=1$ \\
\hline Water intake (8 oz servings) & $8.8 \pm 5.1$ \\
\hline Semesters completed in a bachelors degree & $3.8 \pm 2.9$ \\
\hline Number of credits between 8 and 12 PM & $7.3 \pm 4.3$ \\
\hline Number of credits between 12 and 4 PM & $5 \pm 2.1$ \\
\hline Number of credits between 4 and 8 PM & $2.9 \pm 3.3$ \\
\hline
\end{tabular}

\section{Results}

Demographic data \& methodological efficacy

The sample cohort contained nine full-time college students (five female, four male) with a mean age of $20.2 \pm 1.6$ years, who were diagnosed with a concussion. Demographic characteristics were described in Table 1. For responding to text messages and phone calls, the average response rates for text messages and phone calls were 92 and $93 \%$, respectively. Further segregation of these data can be seen in Figure 2.

\section{Beneficial factors for concussion symptom recovery}

The current study identified longer sleep duration, greater water intake and overall time since injury to be beneficial factors on concussion symptom recovery (Figure 3). Sleep duration was a significant factor altering dizziness with an estimate value of -0.0006 (standard error of mean $[\mathrm{SE}]=0.0002, \mathrm{p}=0.0004$ ), indicating a $0.06 \%$ reduction in symptom severity per minute of sleep acquired over the course of recovery. Water intake was a significant factor in reducing anxiety with an estimated value of $-0.17(\mathrm{SE}=0.05 ; \mathrm{p}=0.006)$, indicating that each $8 \mathrm{oz}$ serving of water consumed over the course of recovery reduced anxiety severity by $17 \%$. Time (per hour) was a beneficial factor in ameliorating headache, dizziness, fatigue and anxiety symptoms with estimate values of -0.0033 ( $\mathrm{SE}=0.0012$; 
(A)

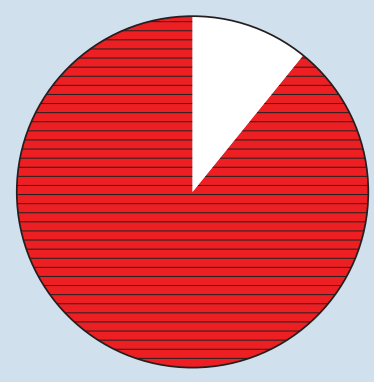

(B)

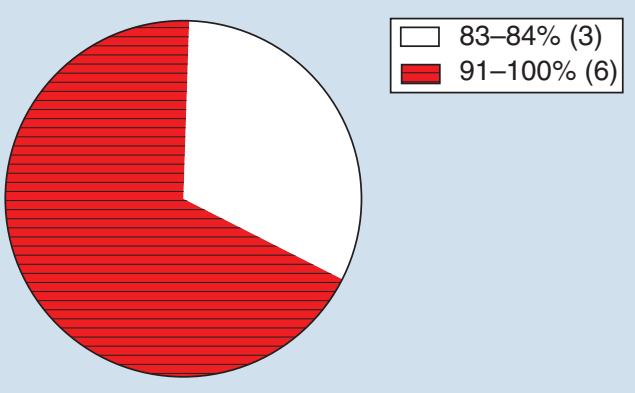

Figure 2. Study procedural compliance. Response rates for phone calls (A) and text messages (B). Individual response rates are represented as part of a whole, separated by percentage ranges with accompanying (n) number of participants within that range.

(A)

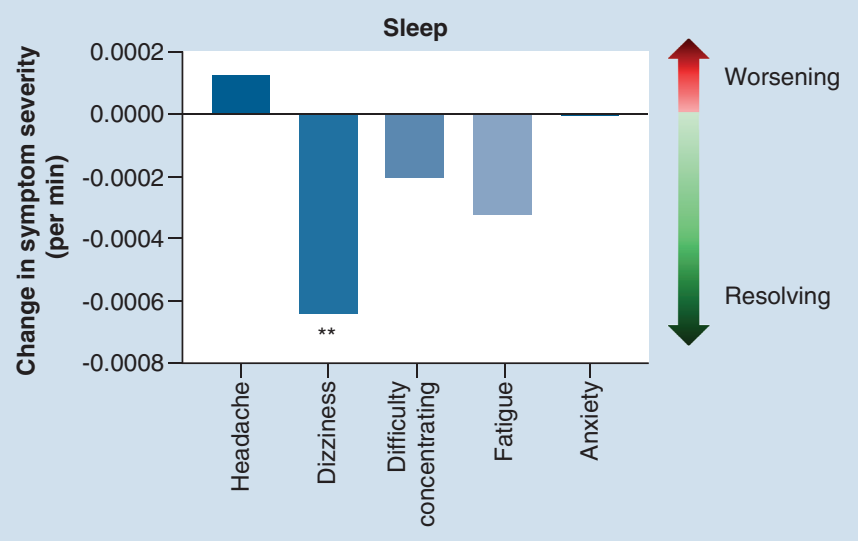

(B)

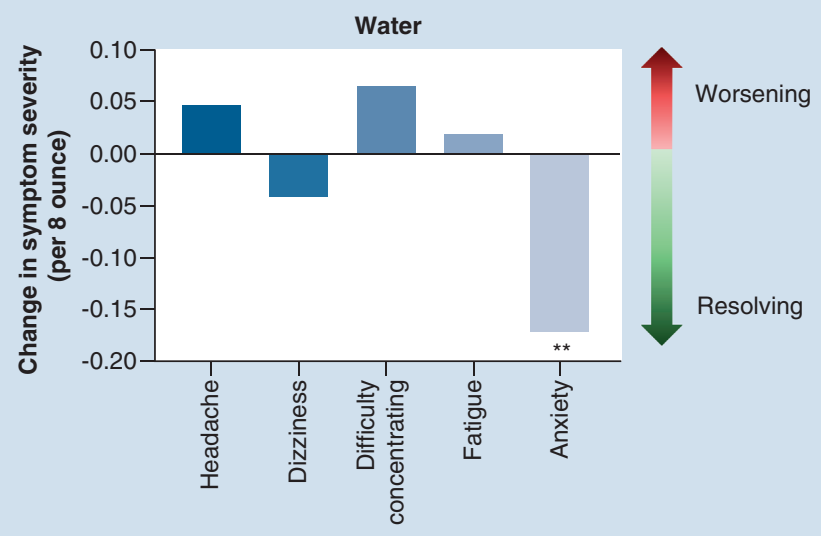

(C)

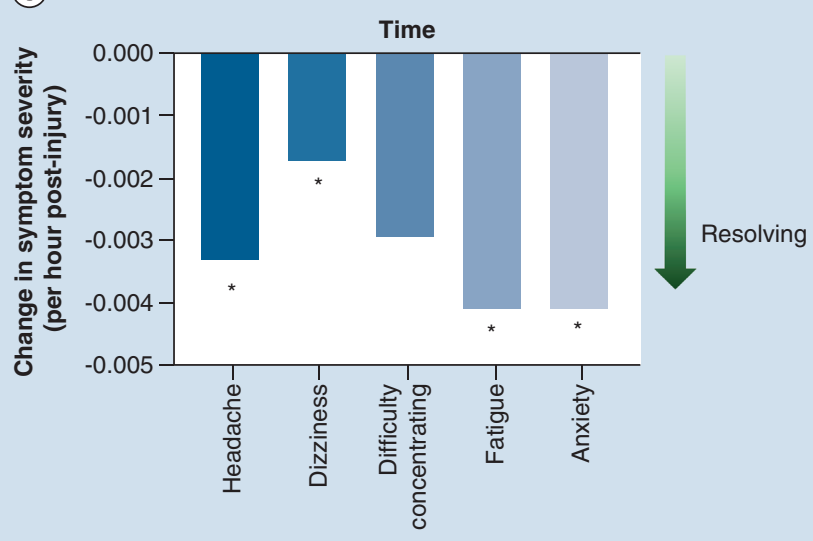

Figure 3. Beneficial factors for concussion recovery. Behavioral variables vs symptom severity. Music vs symptoms (A), and physical activity vs symptoms (B) are shown respectively. Our five text message symptoms are listed along the $\mathrm{x}$-axis, with symptom severity change along the left-sided $y$-axis, and effect of the behavior listed along the right-sided $y$-axis. Significance was set at $p<0.05$. ${ }^{*} \mathrm{p}<0.05 ;{ }^{* *} \mathrm{p}<0.001$. 
(A)

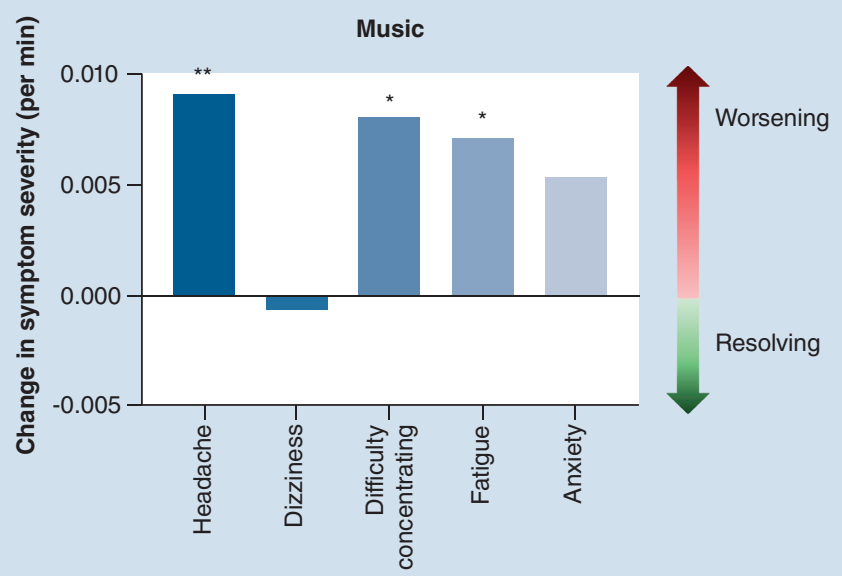

(B)

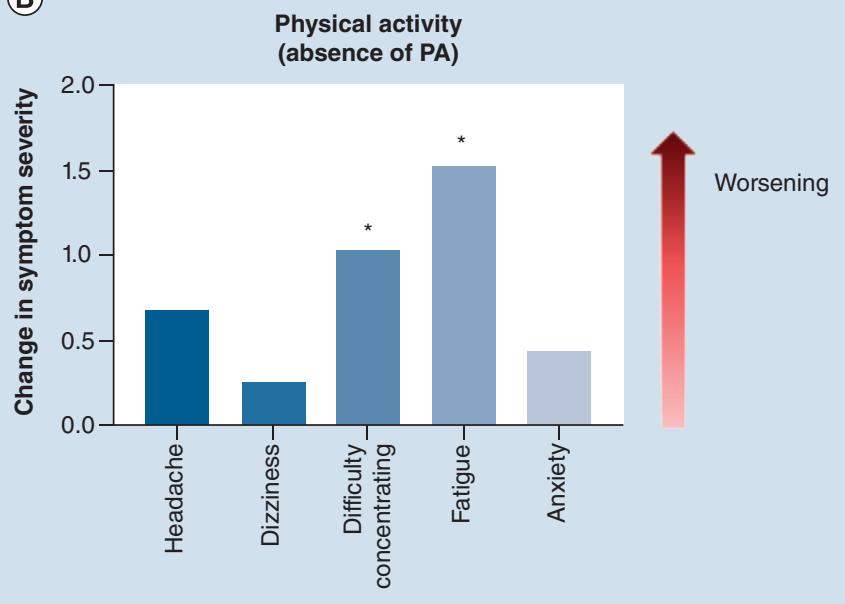

Figure 4. Adverse factors for concussion recovery. Behavioral variables vs symptom severity. Sleep vs symptoms (A), water vs symptoms (B), and time vs symptoms (C) are shown, respectively. Our five text message symptoms are listed along the $\mathrm{x}$-axis, with symptom severity change along the left-sided $y$-axis, and effect of the behavior listed along the right-sided $y$-axis.

${ }^{*} \mathrm{p}<0.05 ;{ }^{*} \mathrm{p}<0.001$.

$\mathrm{p}=0.0064),-0.0017(\mathrm{SE}=0.0007 ; \mathrm{p}=0.0174),-0.004(\mathrm{SE}=0.0014 ; \mathrm{p}=0.0026)$ and $-0.0041(\mathrm{SE}=0.0014$;

$\mathrm{p}=0.0038)$, respectively. Please see Table 2 for a summary of the results.

\section{Adverse factors for concussion symptom recovery}

Longer duration of music and absence of physical activity were shown to exacerbate symptoms (Figure 4). Time spent listening to music produced a milder increase in headache, difficulty concentrating and fatigue, resulting in estimate values of $0.0091(\mathrm{SE}=0.0026 ; \mathrm{p}=0.0006), 0.0081(\mathrm{SE}=0.0033 ; \mathrm{p}=0.014)$ and $0.0072(\mathrm{SE}=0.0030$; $\mathrm{p}=0.016$ ), respectively. These results indicate that music may increase these symptoms by 0.7 to $0.9 \%$ per minute of music listened to. Physical activity was analyzed in a binary fashion (participated or did not participate). Our results show that the absence of physical activity can significantly increase symptoms of difficulty in concentrating and fatigue, resulting in estimate values of $1.035(\mathrm{SE}=0.3852 ; \mathrm{p}=0.036)$ and $1.53(\mathrm{SE}=0.3658 ; \mathrm{p}=0.006)$, respectively, indicating that the absence of physical activity can exacerbate these symptoms by as much as $153 \%$.

\section{Exit survey- student perspectives on recovery}

Noteworthy findings, which were defined as containing at least a third of the sample size, were seen with exit interview questions 1, 2 and 4. In response to the question, "During your recovery, which subject(s) was/were the most difficult?", 33 and 44\% of participants expressed concerns with math and computer use during their recovery, respectively. In response to the question "What modifications were helpful to you during your recovery?", 66, 56 and $33 \%$ of participants expressed that additional time on assignments/exams, reducing screen brightness and wearing sunglasses in class were helpful during recovery, respectively. Additional time on assignments/exams was selected by six participants of which $4 / 6$ were enrolled in math-based courses, 5/6 computer-based courses and 4/6 enrolled in both. Eighty percent of participants who selected reducing screen brightness and $100 \%$ of participants who selected wearing sunglasses were enrolled in computer-based courses. At last, in response to the question "Overall, what made you feel better?", participants found rest/sleep (66\%) and taking breaks from screens, class and homework (44\%) to be beneficial.

\section{Discussion}

The current study identified five variables that produced a significant association with participants' concussion symptoms. Since this study is the first to longitudinally track students' recovery in the context of an academic setting and included a small sample size, we feel that it is premature to draw a causal inference on relationships between the observed behavioral factors and concussion symptom recovery. Therefore, in this section, we will 
introduce the potential mechanisms with which these factors are connected. To begin, long duration of music exposure was shown to have an adverse effect on headache, difficulty concentrating and fatigue. This observation is potentially the result of an over stimulation of cortical tissue with a concurrent reduction in cerebral blood flow supplying metabolic demands, which is supported by the previous studies that showed diminished auditory function and blood flow after a concussion $[27,28]$. Second, our data indicates that the absence of physical activity increased symptoms of fatigue and difficulty concentrating, corroborating the large body of emerging evidence advocating the beneficial effects of physical activity postconcussion. Literature has shown that graded physical activity induces trophic outcomes [29-34], such that when the activity is dosed correctly and in a timely manner, it can ameliorate concussion symptoms $[29,30]$. Therefore, it is plausible that our participants' decision to forgo these advantageous activities may have produced rises in symptom severity. Third, longer overall sleep duration was shown to reduce the severity of dizziness among participants. The health benefits of sleep are widely understood, yet a large percentage of college-aged individuals report being sleep deprived [35,36]. Additionally, sleep deprivation has been shown to dysregulate sympathetic and parasympathetic balance of heart [37-39]. We speculate that when concussive neural damage is coupled with sleep deprivation, there is a possibility to synergistically worsen one's dizziness during the recovery phase. Forth, despite robust statistical significance, the connection between water intake and reduction in anxiety symptoms is difficult to delineate. This relationship, like others listed within this section, require further investigation. At last, time was the final variable to show significant interaction with symptoms. This association was implicit as concussion symptoms are expected to normally resolve within 10-14 days for adults and 1 month for children [34].

Exit survey reports from participants indicate two troublesome academic areas: mathematics and computer usage. The present findings corroborate previous RTL research, identifying math as the most troublesome academic subject [16]. Our results also parallel that of Ransom et al. [16], who reported math being the most difficult subject across multiple academic levels (freshman-junior vs elementary-high school). Functional MRI data shows that postconcussion patients exhibit a decreased blood oxygen level-dependent signal to the posterior cingulate and cerebellum when tasked with the Automated Neuropsychological Assessment Metrics Math Processing subtest, compared with healthy controls [29], indicating that concussive trauma may place a metabolic burden on brain regions that contribute to mathematical processing. Additionally, white matter tract abnormalities of the corpus callosum have been seen via diffusion tensor imaging in concussed pediatric patients and were in conjunction with mathematical deficits [40]. Other diffusion tensor imaging studies have also indicated mathematical performance to be associated with the commissural fibers of the corpus callosum [41], of which are among fibers commonly injured during concussion [42]. Data from both previous RTL literature and imaging studies corroborate our participants' arduous perspectives toward math-based coursework, and perhaps their favorability toward additional time to complete assignments.

Reluctance toward computer usage in the present study also agrees with current literature. The concussion consensus statement most notably recommends screen time be monitored during the first stage of a graduated return-to-school protocol, steadily increasing usage throughout recovery [34]. Seemingly, this recommendation incorporates the understanding that liquid crystal display screens typically refresh 60-times per second [43]. The rapid flickering of these screens is negligible to healthy individuals, however, concussed patients with photosensitivity have find this symptom provoking [43]. This deficit may partly explain why three participants who reported higher severities of sensitivity to light during the initial postconcussion testing also selected computer usage as their most troublesome academic area and why the majority of participants enrolled in computer-based courses selected wearing sunglasses $(80 \%)$ and reducing screen brightness $(100 \%)$ as beneficial behaviors.

There are several strengths of the current study. To our knowledge, this is the first study to not only use text messaging to collect symptom data, but also the first study to collect symptom data as frequently as 4-times per day. The use of daily phone calls, instead of online surveys, to gather comprehensive data from participants is another innovative characteristic. There are two rationales for choosing this method: to limit exposure to electronic screens, as used by other studies [32,44], given that sensitivity to light and computer screens have been known as a chief complain of concussion $[43,45]$, and speaking to a researcher, instead of an automated system, allows participants to ask for clarification, such as confirming that sexual activity is considered physical activity. Finally, requiring a 48-h period of academic participation, following a return to baseline symptoms, allowed us to capture participants' tolerance to a wider range of their course load.

In contrast, the current study is not without limitations. It is important to note that symptom reports through text messages exposed participants to view a screen. There is the potential for this screen exposure to agitate 
symptoms, yet more than $90 \%$ of college-aged individuals use smartphones, making this a highly accessible direct line of communication between researchers and participants. Further rationale behind using text messaging was as follows: responding to symptom questions via text message takes an average of $30 \mathrm{~s}$, smartphones have voice-to-text capability, allowing participants the option to vocalize their answers instead of typing and not all students have access to a personal computer for online symptom surveys. Therefore, text messaging was thought to be brief, highly effective and feasible method for repeated data collection.

The small sample size limits not only the breadth of academic majors that were included, but the various types of courses that students may be enrolled in and the level of study (undergraduate vs graduate). Additionally, a small sample size inherently limits the generalizability of the results, thus our findings are preliminary at best. Second, our criteria for recovery required a return to baseline symptomology for eight consecutive text messages, as well as 2 days of academic participation. These criteria have two limitations, the consecutive requirement of the text messages, and classes are not held on weekends which may separate the 2 days of academic participation; delaying 'recovered' status. Third, in an effort to minimize the time needed to answer our text messages (exposure to screens), we intentionally limited our list of symptoms to five. Unfortunately, this required us to exclude other symptoms such as sensitivity to light, sensitivity to noise and fogginess, which may have been associated with our independent variables as well. Forth, to keep our diagnosing physician unbiased in their practice, we did not track their medical recommendations or provider referrals for patients (e.g., abstinence from homework, screens, medication prescriptions, physical therapy referral, etc.), potentially influencing participant behavior.

Larger bodies of evidence-based investigations will be needed to establish a guideline that can be used in various academic settings. Until now, RTL has lacked a methodological approach to gathering such evidence-based data, therefore, we recommend researchers reproduce this study in a larger scale and at various levels of academia. A personalized concussion recovery protocol in the academic setting is the ultimate goal, but it is important to first understand the general behavioral pattern in respect to concussion recovery. Eventually, these behavior-symptom data are intended to provide practitioners with evidence-based guidance for their concussion treatments. The relationships identified in the current study are hopeful, however, we noticed that participants' recovery trajectory and lifestyles were largely heterogeneous. Practitioners should remain cognizant of this limitation as they read this paper.

\section{Conclusion}

The present study multimodally collected data on several variables, both independent and dependent, in a longitudinal fashion. The data provides evidence that ample sleep duration, sufficient water intake and overall time have beneficial effects on concussion symptoms during one's recovery, whereas long duration of music exposure and lack of physical activity may exacerbate concussion symptoms over time. These associations, however, must be investigated in a larger sample size if they are to be substantiated beyond early recommendations that are of profound importance to concussion care in the academic settings.

\section{Future perspective}

Our findings introduce a novel approach to monitoring concussed students throughout their recovery. Because this evidence is the first of its kind, a large-scale exploration must be done to validate the preliminary theories. Given the robust methodology of the current study, we speculate that this will be adopted and improved upon by others, lending greater insight to establishment of RTL. It is prudent to allow the following questions to guide us: How can we make academic instructors and administrators aware of current practices for concussion management? How do these prescribed behavior modifications translate into academic accommodations? How can medical and academic personnel work together to ensure a seamless recovery for their patients/students?

Supplementary data

To view the supplementary data that accompany this paper please visit the journal website at: www.futuremedicine.com/doi/full/10.2217/cnc-2019-0004 
data; preparation, review, or approval of the manuscript; or decision to submit the manuscript for publication. The authors have no other relevant affiliations or financial involvement with any organization or entity with a financial interest in or financial conflict with the subject matter or materials discussed in the manuscript apart from those disclosed.

No writing assistance was utilized in the production of this manuscript.

Ethical conduct of research

The authors state that they have obtained appropriate institutional review board approval or have followed the principles outlined in the Declaration of Helsinki for all human or animal experimental investigations. In addition, for investigations involving human subjects, informed consent has been obtained from the participants involved

Open access

This work is licensed under the Creative Commons Attribution4.0 License. To view a copy of this license, visit http://creativecommons.org/licenses/by/4.0/

\section{Summary points}

Background

- Return-to-learn (RTL) has appeared to be a secondary focus in comparison with the abundantly researched return-to-play protocols implemented ubiquitously.

- Limited studies have examined the RTL aspect of concussion recovery and the effects premature classroom attendance may cause.

- No evidence-based data has been offered regarding RTL.

Methodology

- Daily text message and phone calls were used to longitudinally track students, both yielding exceptional response rates.

- This is the first study to sample symptom severity data four-times per day.

- Accelerometer data were used to accurately quantify step count and sleep, versus traditional recall methods.

Behavior-symptom associations

- Listening to music was associated with increased headache, difficulty concentrating and fatigue.

- Absence of physical activity was associated with increased difficulty concentrating and fatigue.

- Greater water intake was associated with decreased anxiety.

- Greater amounts of sleep were associated with decreased dizziness.

- Time postinjury was associated with a decrease in all symptoms, with the exception of difficulty concentrating.

Student perceptions

- Math and computer-oriented courses were reported as the most difficult courses.

- Additional time on assignments/exams and reducing screen brightness were reported as the most beneficial accommodations.

Future directions

- The current study should be reproduced in a larger scale, and at various levels of academic settings.

- As RTL research evolves, researchers should allow the following questions to guide their exploration: When is it effective, and or safe, to implement a RTL protocol? How can student-athletes complete a RTL protocol in tandem with a return-to-play protocol? How will we include academic instructors and administrators in this process?

\section{References}

1. Friedersdorf C. 10 Ideas That Changed the World in 2012. The Atlantic, DC, USA (2012). https://www.theatlantic.com/international/archive/2012/12/10-ideas-that-changed-the-world-in-2012/266219/

2. Ward J, Williams J, Manchester S. 110 NFL Brains. The New York Times, NY, USA (2017). https://www.nytimes.com/interactive/2017/07/25/sports/football/nfl-cte.html

3. Broglio SP, Cantu RC, Gioia GA et al. National Athletic Trainers' Association position statement: management of sport concussion. J. Athl. Train.49(2), 245-265 (2014).

4. Baker JG, Leddy JJ, Darling SR et al. Factors associated with problems for adolescents returning to the classroom after sport-related concussion. Clin. Pediatr. (Phila.)54(10), 961-968 (2015).

5. Davies S, Coxe K, Harvey HH, Singichetti B, Guo J, Yang J. Qualitative evaluation of high school implementation strategies for youth sports concussion laws. J. Athl. Train.53(9), 873-879 (2018).

6. Kostyun RO, Hafeez I. Protracted recovery from a concussion: a focus on gender and treatment interventions in an adolescent population. Sports Health 7(1), 52-57 (2015). 
7. McGrath N. Supporting the student-athlete's return to the classroom after a sport-related concussion. J. Athl. Train.45(5), 492-498 (2010).

8. Moor HM, Eisenhauer RC, Killian KD et al. The relationship between adherence behaviors and recovery time in adolescents after a sports-related concussion: an observational study. Int. J. Sports Phys. Ther.10(2), 225-233 (2015).

9. Purcell LK, Davis GA, Gioia GA. What factors must be considered in 'return to school' following concussion and what strategies or accommodations should be followed? A systematic review. Br. J. Sports Med.53(4), 250 (2019).

10. Upchurch C, Morgan CD, Umfress A, Yang G, Riederer MF. Discharge instructions for youth sports-related concussions in the emergency department, 2004 to 2012. Clin J. Sport Med.25(3), 297-299 (2015).

11. Wasserman EB, Bazarian JJ, Mapstone M, Block R, van Wijngaarden E. Academic dysfunction after a concussion among US high school and college students. Am. J. Public Health 106(7), 1247-1253 (2016).

12. Weber ML, Welch Bacon CE, McLeod TV. School nurses' management and collaborative practices for student-athletes following sport-related concussion. J. Sch. Nurs. 35(5), 378-387 2018).

13. Weber ML, Welch CE, Parsons JT, Valovich McLeod TC. School nurses' familiarity and perceptions of academic accommodations for student-athletes following sport-related concussion. J. Sch. Nurs.31(2), 146-154 (2015).

14. Williams RM, Welch CE, Parsons JT, McLeod TC. Athletic trainers' familiarity with and perceptions of academic accommodations in secondary school athletes after sport-related concussion. J. Athl. Train.50(3), 262-269 (2015).

15. Williamson CL, Norte GE, Broshek DK, Hart JM, Resch JE. Return to learn after sport-related concussion: a survey of secondary school and collegiate athletic trainers. J. Athl. Train.53(10), 990-1003 (2018).

16. Ransom DM, Vaughan CG, Pratson L, Sady MD, McGill CA, Gioia GA. Academic effects of concussion in children and adolescents. Pediatrics 135(6), 1043-1050 (2015).

17. Carson JD, Lawrence DW, Kraft SA et al. Premature return to play and return to learn after a sport-related concussion: physician's chart review. Can. Fam. Physician.60(6), e310, e312-5 (2014).

18. Halstead ME, McAvoy K, Devore CD et al. Returning to learning following a concussion. Pediatrics 132(5), $948-957$ (2013).

19. O'Neill JA, Cox MK, Clay OJ et al. A review of the literature on pediatric concussions and return-to-learn (RTL): implications for RTL policy, research, and practice. Rehabil. Psychol.62(3), 300-323 (2017).

20. National Conference of State Legislatures. Return-to-learn state laws for students with traumatic brain injuries. (2017) http://www.ncsl.org/research/health/-return-to-learn-state-laws-for-students-with-traumatic-brain-injuries.aspx

21. Sadeh A, Sharkey KM, Carskadon MA. Activity-based sleep-wake identification: an empirical test of methodological issues. Sleep 17(3), 201-207 (1994).

22. de Souza L, Benedito-Silva AA, Pires ML, Poyares D, Tufik S, Calil HM. Further validation of actigraphy for sleep studies. Sleep 26(1), 81-85 (2003).

23. Rose SC, Weber KD, Collen JB, Heyer GL. The diagnosis and management of concussion in children and adolescents. Pediatr. Neurol.53(2), 108-118 (2015).

24. Macartney G, Simoncic V, Goulet K, Aglipay M. Concussion symptom prevalence, severity and trajectory: implications for nursing practice. J. Pediatr. Nurs.40, 58-62 (2018).

25. Bandelow B, Michaelis S. Epidemiology of anxiety disorders in the 21st century. Dialogues Clin. Neurosci.17(3), 327-335 (2015).

26. Haider MN, Leddy JJ, Pavlesen $S$ et al. A systematic review of criteria used to define recovery from sport-related concussion in youth athletes. Br. J. Sports Med.52(18), 1179-1190 (2018).

27. Kraus N, Thompson EC, Krizman J, Cook K, White-Schwoch T, LaBella CR. Auditory biological marker of concussion in children. Sci. Rep.6, 39009 (2016).

28. Yang Z, Yeo RA, Pena A et al. An FMRI study of auditory orienting and inhibition of return in pediatric mild traumatic brain injury. $J$. Neurotrauma.29(12), 2124-2136 (2012).

29. Leddy JJ, Cox JL, Baker JG et al. Exercise treatment for postconcussion syndrome: a pilot study of changes in functional magnetic resonance imaging activation, physiology, and symptoms. J. Head Trauma Rehabil.28(4), 241-249 (2013).

30. Leddy JJ, Haider MN, Ellis M, Willer BS. Exercise is medicine for concussion. Curr. Sports Med. Rep.17(8), 262-270 (2018).

31. Leddy JJ, Haider MN, Ellis MJ et al. Early subthreshold aerobic exercise for sport-related concussion: a randomized clinical trial. JAMA Pediatr. 173(4), 319-325 (2019).

32. Leddy JJ, Haider MN, Hinds AL, Darling S, Willer BS. A preliminary study of the effect of early aerobic exercise treatment for sport-related concussion in males. Clin. J. Sport Med.(2018). doi:10.1097/JSM.0000000000000663 (Epub ahead of print)

33. Leddy JJ, Kozlowski K, Donnelly JP, Pendergast DR, Epstein LH, Willer B. A preliminary study of subsymptom threshold exercise training for refractory post-concussion syndrome. Clin. J. Sport Med.20(1), 21-27 (2010).

34. McCrory P, Meeuwisse W, Dvořák J et al. Consensus statement on concussion in sport-the 5 (th) international conference on concussion in sport held in Berlin, October 2016. Br. J. Sports Med.51(11), 838-847 (2017). 
35. Al Khatib HK, Harding SV, Darzi J, Pot GK. The effects of partial sleep deprivation on energy balance: a systematic review and meta-analysis. Eur. J. Clin. Nutr.71(5), 614-624 (2017).

36. Hershner SD, Chervin RD. Causes and consequences of sleepiness among college students. Nat. Sci. Sleep 6, 73-84 (2014).

37. Franzen PL, Gianaros PJ, Marsland AL et al. Cardiovascular reactivity to acute psychological stress following sleep deprivation. Psychosom. Med.73(8), 679-682 (2011).

38. Tobaldini E, Nobili L, Strada S, Casali KR, Braghiroli A, Montano N. Heart rate variability in normal and pathological sleep. Front. Physiol.4, 294 (2013).

39. Zhong X, Hilton HJ, Gates GJ et al. Increased sympathetic and decreased parasympathetic cardiovascular modulation in normal humans with acute sleep deprivation. J. Appl. Physiol. (1985).98(6), 2024-2032 (2005).

40. Van Beek L, Vanderauwera J, Ghesquière P, Lagae L, De Smedt B. Longitudinal changes in mathematical abilities and white matter following paediatric mild traumatic brain injury. Brain Inj.29(13-14), 1701-1710 (2015).

41. Matejko AA, Ansari D. Drawing connections between white matter and numerical and mathematical cognition: a literature review. Neurosci. Biobehav. Rev.48, 35-52 (2015).

42. Niogi SN, Mukherjee P. Diffusion tensor imaging of mild traumatic brain injury. J. Head Trauma Rehabil.25(4), 241-255 (2010).

43. Mansur A, Hauer TM, Hussain MW et al. A nonliquid crystal display screen computer for treatment of photosensitivity and computer screen intolerance in post-concussion syndrome. J. Neurotrauma.35(16), 1886-1894 (2018).

44. Leddy JJ, Hinds AL, Miecznikowski J et al. Safety and prognostic utility of provocative exercise testing in acutely concussed adolescents: a randomized trial. Clin. J. Sport Med.28(1), 13-20 (2018).

45. Mares C, Dagher JH, Harissi-Dagher M. Narrative review of the pathophysiology of headaches and photosensitivity in mild traumatic brain injury and concussion. Can. J. Neurol. Sci.46(1), 14-22 (2019). 